\title{
Swiss Federation of Clinical Neuro-Societies SFCNS
}

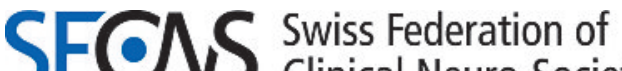 Clinical Neuro-Societies}

Claudio Bassetti

Pr Dr méd.,

président de la SFCNS

Cela fait un peu plus d'un an que la Swiss Federation of Clinical Neuro-Societies SFCNS a été créée. La fédération regroupe les six plus grandes sociétés de médecins en Suisse de neurologie et représentant plus de 800 membres actifs. Elle fut fondée en juin 2008 sous la houlette du Pr Claudio Bassetti (Président de la Société Suisse de Neurologie, SSN), du Pr Gerhardt Hildebrandt (ancien président de la Société Suisse de Neurochirurgie, SSN) et du Pr Anton Valavanis (Président de la Société Suisse de Neuroradiologie, SSNR) afin de développer conjointement les intérêts de la neuroscience clinique en Suisse. Deux ans auparavant, les membres fondateurs avaient en collaboration avec le Pr Christian Hess, ancien président de la SSN, posé la première pierre de la SFCNS à Zurich.

\section{La fédération regroupe les six plus grandes sociétés de médecins en Suisse de neurologie}

Correspondance:

Secrétariat administratif de la SFCNS

Gabriela Deutsch c/o IMK

Institut pour la médecine

et la communication SA

Münsterberg 1

CH-4001 Bâle

Tél. 0612713551

Fax 0612713338

sfons@imk.ch
La SFCNS réunit actuellement les six sociétés fondatrices suivantes:

- Société Suisse de Neurologie, SSN

- Société Suisse de Neurochirurgie, SSN

- Société Suisse de Neuroradiologie, SSNR

- Société Suisse de Neurophysiologie Clinique, SSNC

- Société Suisse de Neuropédiatrie, SSNP

- Société Suisse de Neuropathologie, SSNPath

Les objectifs de la SFCNS sont les suivants:

- Promouvoir les neurosciences cliniques en Suisse

- Promouvoir la collaboration interdisciplinaire avec les organisations neuroscientifiques (organisations professionnelles, instances politiques et porteurs des coûts) et enfin la collaboration avec d'autres sociétés neuroscientifiques intéressées

- Promouvoir la formation continue et post-graduée dans le domaine des neurosciences de manière générale

- Organiser les formations continues et postgraduées.

\section{Organisation au sein de la SFCNS}

Étant donné que la SFCNS ne permet pas d'adhésion individuelle mais uniquement des adhésions collectives, les diverses sociétés de médecins précitées sont représentées par des délégués. Deux délégués sont autorisés par société membre, le/la président(e) (ou représentant) de la société étant automatiquement élu comme délégué(e). La mission de l'assemblée des délégués en tant qu'organe supérieur de la fédération consiste notamment à élire son propre comité, constitué d'un président, un vice-président et un secrétaire/ trésorier ainsi que deux assesseurs. Les premières élections se sont tenues en 2009 pour la durée de mandat de 2009 à 2011; ce faisant, il faut veiller à représenter les différents groupements d'intérêts et différentes disciplines.

\section{First SFCNS Congress en 2010 à Bâle}

La SFCNS prévoit, tous les trois ans, la tenue d'un congrès. En tant que première, il s'agit pour le «First SFCNS Congress», qui se tiendra du 2 au 4 juin 2010 à Bâle, d'une première étape importante dans l'atteinte des objectifs de la société. Sur le plan du contenu, le congrès sera consacré aux maladies cardiovasculaires dans le cadre de la campagne de la SFCNS sur les AVC (SFCNS stroke campaign 2010). L'épilepsie, la colonne vertébrale et la moelle épinière constitueront également d'autres thèmes clés. Grâce à un programme attrayant la grande majorité des sociétés conviées devraient se sentir concernées, si bien que nous attendons près de 700 participants de Suisse et des pays voisins lors de cette manifestation. Lors du congrès, différents prix de recherche seront décernés, notamment pour la première fois, la récompense très lucrative du SFCNS Award.

\section{Autres sociétés}

La SFCNS est ouverte à de nouvelles adhésions de sociétés ou de groupements d'intérêts. Pour tout renseignement complémentaire, veuillez vous rendre sous www.sfcns.ch ainsi que sur le site web du congrès sous www.imk.ch/sfcns 2010 ou bien contactez directement notre secrétariat administratif. 